\title{
Passenger Control in Smart Cities Using Deep Learning
}

\author{
Delna T D, Dhanya P Pauly, Dona Johnson, Jesta Jose \\ Department of Computer Science and Engineering \\ Sahrdaya College of Engineering and Technology Kodakara Thrissur \\ APJ Abdul Kalam Technological University
}

\begin{abstract}
In the current smart city background, people are facing a lot of accidents at the major traffic points of the business towns due to growing population and vehicles growth in smart and metropolitan cities.In this method we consider the auto taxies as well as the public transport. We know that due to the overload in the vehicles the accidents are increasing day by day so using this method the number of accidents be able to be avoided or reduced. This system is introducing the deep learning approach to find the overload in vehicles. We are considering the luggage that is taken along with the passenger and an average weight is given for the load. Then it is combined with the number of passenger and system will predict whether the vehicle is overload or not. Mainly because of using deep learning concepts we can increase the speed of the process and the efficiency. The system will analyse the number of passengers using real time videos using camera and system detect and compare with the overloading conditions to avoidaccidents.
\end{abstract}

\section{INTRODUCTION}

This section gives an overview about the objective of the project. System predicts the number of passengers in the vehicle and the load. It detects the number of passenger in the vehicle using deep learning. Number of passengers beyond a limit is not encouraged to travel in the vehicle. The passenger control in smart cities is planning of avoiding overload in taxies. Proposed system provides the detection of passenger overload in taxies. Overload can be detected by using passenger detection method. This is one of the best methods to detect overload in auto taxies. In this we consider the auto taxies as well as the public transport. Setting the limit for passengers according to capacity of the vehicle. Number of passengers will be counted and also it will detect the back bag, luggage and school bag .A deep learning system will be provided in the corresponding vehicle with camera. The system is introducing and the deep learning approach to find the overload in vehicle. Deep learning is a subset of machine learning where artificial neural networks, human brain-inspired algorithms, learn from large quantities of data. Deep learning helps systems to solve complex problems, even though they use a very diverse, unstructured and interconnected data set. It will give a warning if there is overloading taxies. Through this number of accidents due to overload can be avoided. Camera is used to acquire the real time video inside the vehicle and system detects and analyzes the overload using deep learning mechanism. Face detecting whether the face is of the human and it will divide the passengers as single blocks and then count the number of blocks. The number of blocks is equivalent to the count of passengers and is not effective for the vehicle's overload detection. So we find the luggage that is taken along with the passenger and an average weight for the load is given. This is then paired with the number of passengers, and the system can determine whether or not the car isoverloaded.

\section{DRAWBACKS OF EXISTINGSYSTEM}

Passenger overload in public transport lead to many accidents. The existing system detects the load in the vehicle but it is not predicting whether it is human overload or the load in the vehicle. The suspension system of the vehicle will transmit forces and torques that work between the wheel and the frame, and cushion the impact force transmitted from the rough ground surface to the frame or body to absorb the energy produced by impact. Changes in vehicle load can cause the elastic element to deform, and the vehicle body will have a vertical displacement. Therefore different vehicle loads correspond to a certain distance between the characteristic points of the vehicle body and the road surface under static conditions. When there is an input of a vehicle vibration excitation, a vibration response is produced by the suspension of the vehicle, and the distance from the characteristic point on the body of the vehicle to the ground periodically changes. Method of identification of characteristic distance dependent on load value. You can measure the bearing properties of the front suspension and the rear suspension. Through the visual roadbed the characteristic distance of the front and rear suspensions can be obtained and also the static deflection value is obtained.

Therefore, the major drawbacks of the existing system are

$>$ Only concerns about the load of thevehicle.

$>$ Not predicting the passenger count andoverall weight.

$>$ Cannot be used in public transportfor passenger safety.

$>$ Accuracy in weight isless.

$>$ Lack of knowledge about the number ofpassengers 


\section{METHOD}

The program that we are implementing makes use of different domains, namely machine learning. The entire system can be loosely classified as two partsi.e. the part of detection and the part of classification. The proposed program provides for passenger overload detection in taxis. Use of passenger detection method to detect overload.In this method we find car taxis as well as public transport. Setting the limit for passengers according to the vehicle's capacity. The number of passengers will be counted and the back seat, luggage and school bag will also be detected. A deep learning system will be equipped with camera in the corresponding vehicle.The program is being implemented and a deep learning approach to detecting the vehicle overload. It will offer a alert if there is overload in taxis. Using camera, acquire real time video by detecting and analyzing the overload using deep learning mechanism. One camera at front and other at top.Face detecting if the face is individual, splitting the passengers as single blocks and counting the number of blocks afterwards. The number of blocks is equal to the number of passengers it is not effective for the vehicle's overload detection. We consider the luggage that is taken along with the passenger and an average weight for the load is given. This is then paired with the number of passengers, and the device will determine whether or not the vehicle is overloaded.

Count the number of passengers by camera according to the real-time video and compare it to the data in the database. In the analysis process, the number of passengers is compared to the system cap. Changes in vehicle load will cause the elastic element to deform, the vehicle body will displace in the vertical direction. Different vehicle loads lead to a certain distance between the characteristic points of the vehicle body and the road surface under static conditions. When there is an input of a vehicle vibration excitation, a vibration response is produced by the vehicle suspension, and the distance from the characteristic point on the body of the vehicle to the ground constantly changes weight.If the number of people traveling on holidays is higher, the problem of congestion can be easily induced. After classification of the classifier, YOLOv3 method predicts multiple detection boxes and many of them overlap with other boxes across a wide area. Each box of candidates has a score of confidence which represents how sure the model is that the box contains an item. These studies take advantage of computer vision and do all the jobs of detecting door switches, detecting floors and detecting passengers by using only the camera sensor, and achieve the goal of real detection of passenger flow statistics. Passenger flows are counted via camera in this method, and the loads are also measured and predict whether or not YOLOv3 method overloads passengers.

The standard form of vehicle overload identification is the establishment of detection points on highways. With this method, the precise definition of whether it is overloaded is difficult. Set detector device can be correctly mounted Identify vehicle weight but installation is complicated and difficult to facilitate on a wide scale.
With the continuous growth of the transport industry, the issue of overloading vehicles is becoming increasingly severe. Vehicle overload severely damages road facilities and causes road traffic accidents which pose a threat to the lives and safety of people. Vehicle balance system is based on capacitive sensor. When the capacitive sensor senses the load of the vehicle, the vehicle's impact on the ground shifts the distance between the capacitive sensor's two surfaces, leading to a shift in capacitance. The balance of the vehicles is bulky, expensive, and destructive to the road and low vehicle load efficiencyDetection of passenger overload in taxis based on deep learning is unsupervised learning by predicting unstructured or unlabeled data using unsupervised learning. It's a subset of machine learning where artificial neural networks, human brain-inspired algorithms, learn from large quantities of data.Deep learning enables machines to solve complex problems, even when using a very diverse, unstructured and interconnected data set

Fig 1 Displays an architectural description of the system proposed. In this initially a prediction of passenger over load using deep learning in car.

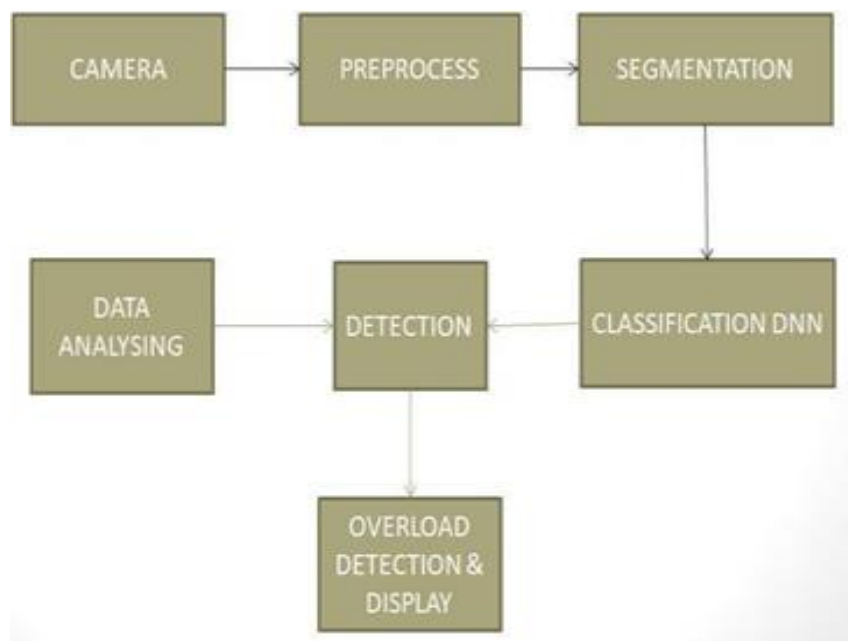

Fig 1:- Architectural Diagram

The various passenger control system modules include camera, pre-processing, segmentation, DNN classification, detection and display, and data analysis. The following brief descriptions are given about these modules:

\section{A. Camera}

Using camera to acquire image. One in front camera. And another upstairs. Here it was used to capture passenger's real time video. A camera is an optical instrument used for image recording. Cameras are sealed boxes (the camera body) with a small hole (the aperture) at their most basic, that let light in to capture an image on a light-sensitive surface.

\section{B. Pre-process}

There are few preprocessing like noise calculation, gray scale conversion, threshold and canny. 


\section{Noise Calculation:}

Sense to suppress the noise on pre-processing data. Noise to be avoided to reduce the complexity in experiment. Real-world data, which is the input of the system, are affected by several components among them, the presence of noise is a key factor. The presence of noise in the data may affect the intrinsic characteristics of a classification problem, since these corruptions could introduce new properties in the problemdomain.

\section{Drayscale Conversion:}

Conversion of color image into grayscale image. It reduces a lot of information which are not required and also will save lot of computational power as well. It is a image one in which each pixel value is a single image sample representing only an amount of light which carries only intensity information. This images is a kind of black-andwhite or gray monochrome, are composed exclusively of shades of gray. In this experiment no need of color image.

\section{Threshold:}

Threshold image analysis technique is a type of image segmentation that isolates objects by converting grayscale images into binary images. So that the number of segments can be reduced.

\section{Canny:}

The Canny edge detector is an edge detection operator that detect a wide range of edges in images using a multistage algorithm.

It has three adjustable parameters like the width of the Gaussian noise and the low and high threshold for the hysteresis thresholding.

\section{Segmentation}

Segmentation is the process of portioning a digital images into multiple segments. The segmentation process is to simplify or change the representation into something that is more meaningful, easier to analyze and understand. Image segmentation is typically used to locate the objects and boundaries (lines, curves, etc.) in images. More precisely, the process image segmentation is of assigning a label to the every pixel in an image such that pixels with the same label will share certain points. Contouring is the important part in segmentation.

\section{$>$ Contouring:}

Contours can be explained simply as a curve joining all the continuous points, having same colour or intensity. The contouring is a useful tool for shape analysis, object detection and recognition.

\section{Classification DNN}

Classifies the images into person, handbag and suitcase using Deep Neural Network. Train a simple neural network in order to perform the regression and classification. It is able to improve on performance of DNN by Adding more layers, experimenting different optimizer function/regularization.

\section{E. Detection and Display}

Number of passenger's detected then average weight is detected and displayed on the screen. Here the number of passengers counted and also weight detected by analyse the luggage and then it is displayed on the screen.

\section{F. Data Analysing}

Data analysing is a process for obtaining raw data and converting it into useful information for decision making by users. Data analysis is defined as a process of cleaning transforming and modelling data in order to discover useful information for decision making in business purposes. An analyzer or analyzer is a person or luggage that analyses given data. It also examines in detail the structure of the given data and tries to find patterns and relationships between parts of the data. An analyzer can be a piece of hardware or a computer program running on acomputer.

Fig 2 shows the use case diagram of the proposed system in which the user can obtain the output display. Such that overload detector will be able to predict the overload.

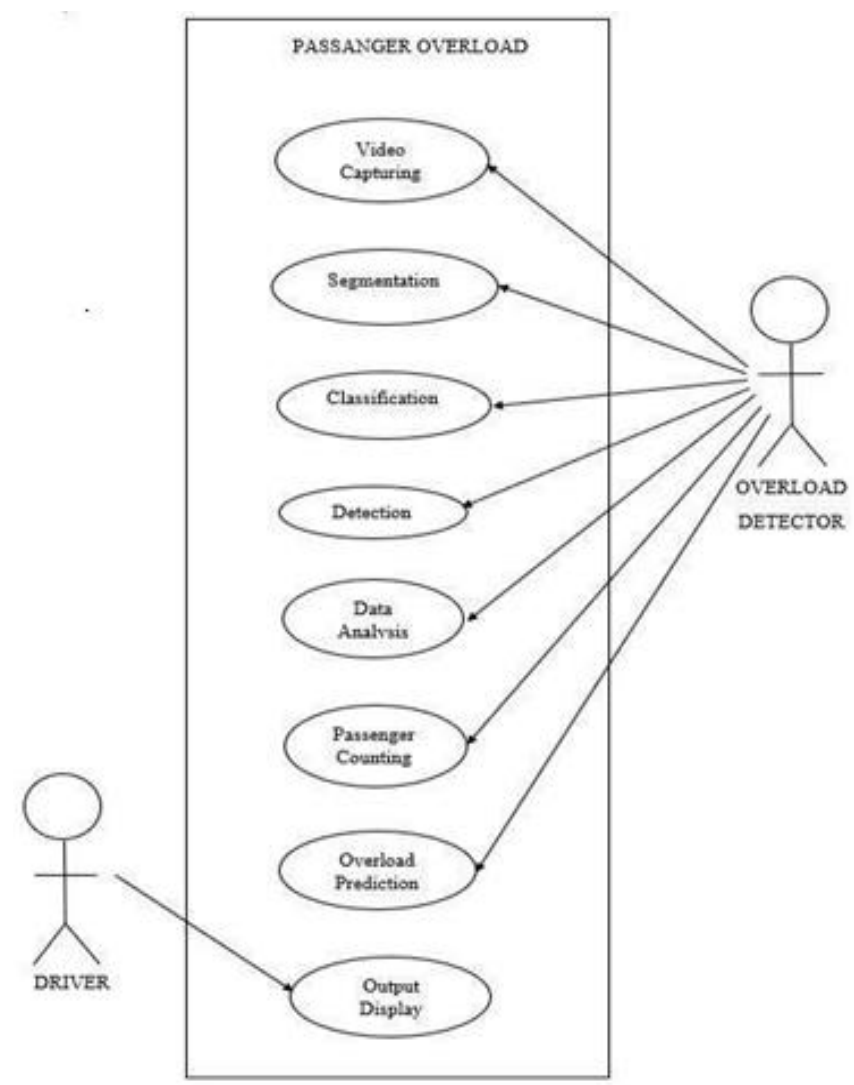

Fig 2:- Use Case Diagram

The processes involved here is such as video capturing, segmentation it is the process of partitioning a digital image into multiple segments, Then it will classifies person, handbag and suitcase using deep learning technology. Next it's carry out for detecting average count of passengers and it will be displayed on the screen. 


\section{RESULTS}

The passenger control in smart cities using deep learning solve the problem of overload in the vehicles by counting each people or bags. Using this system has achieved good results for detecting overloading in vehicles. Number of passengers collisions due to the overloaded problem is expected to be reduced using this method.

While travelling, the real time video was successfully captured by the attached camera module. Face detecting whether the face is of the human and it will divide the passengers as single blocks and then count the number of blocks. The number of blocks are equal to number of passengers it is no efficient for overload detection of the vehicle. So we are considering the luggage that is taken along with the passenger and an average weight is given for the load. Then it is combined with the number of passenger and system will predict whether the vehicle is overload or not. For detection part we got an accuracy of $90 \%$ and the classification portion showed a $97 \%$ accuracy.

\section{CONCLUSION}

System will provide the detection of passenger overload in taxies. Overload can be detected by using passenger detection method. In this method we consider the auto taxies as well as the public transport. We know that due to the overload in the vehicles the accidents are increasing day by day so using this method the number of accidents can be avoided or reduced. In this method the number of passengers will be counted and also it will detect the back bag, luggage and school bag. A deep learning system will be provided in the corresponding vehicle with camera. The system is introducing and a deep learning approach to find the overload in vehicle .It will give a warning if there is overload in taxies. Acquire the real time video using camera and system detect and analyze overload using deep learning mechanism using camera. So we are considering the luggage that is taken along with the passenger and an average weight is given for the load. Mainly because of using deep learning concepts we can increase the speed of the process and the efficiency .So using this method we can detect the overload in the vehicles and it can be solved veryeasily.

\section{ACKNOWLEDGEMENT}

We would like to express our immense gratitude and profound thanks to all those who helped us to make this project a great success. We express our gratitude to the almighty God for all the blessings endowed on us. We express our thanks to our Executive Director REV.FR. GEORGE PAREMAN, Director Dr. ELIZABETHELIAS.

Principal Dr. NIXON KURUVILA for providing us with such a great opportunity. We are thankful for the help and appreciation we received from head of thedepartment Dr. RAJESWARI M., project coordinators Ms. DEEPA DEVASSY, Ms. ANLY ANTONY and Mr. WILLSON JOSEPH. We would also extend our deep sense of gratitude to our project guide Ms. SHEETHAL M S for their guidance and advice. We would like to express our gratitude towards our parents for their timely co-operation and encouragement. Every project is successful due to the effort of many people. Our thanks and appreciations go to all our peers who had given us their valuable advice and support and pushed us into successfully completing thisproject.

\section{REFERENCES}

[1]. A. S. A. Nasir, N. K. A. Gharib and H. Jaafar, "Automatic Passenger Counting System Using Image Processing Based on Skin Colour Detection Approach," 2018 International Conference on Computational Approach in Smart Systems Design and Applications (ICASSDA), Kuching, 2018, pp. 18, doi: 10.1109/ICASSDA.2018.8477628.

[2]. Q. Fan, "Overload Monitoring System for Inland River Ferries," 2019 5th International Conference on Transportation Information and Safety (ICTIS), Liverpool, United Kingdom, 2019, pp. 1487-1490, doi: 10.1109/ICTIS.2019.8883679.

[3]. Z. He, Y. Chen, L. Sun, J. Zhong and Y. Zhu, "Identifying Station-Link Correlation for Target Passenger Flow Control in Subway Network," 2018 21st International Conference on Intelligent Transportation Systems (ITSC), Maui, HI, 2018, pp. 84-89, doi:10.1109/ITSC.2018.8569533.

[4]. Z. Sheng, K. Tian, Q. Tian and H. Qu, "A Faster RCNN Based High-Normalization Sample Calibration Method for Dense Subway Passenger Flow Detection," 2018 11th International Congress on Image and Signal Processing, BioMedical Engineering and Informatics (CISP-BMEI), Beijing, China, 2018, pp. 1-5, doi: 10.1109/CISP-BMEI.2018.8633050. 